\title{
The first injection in a human being of macromolecules whose primary structure was developed from a religious text
}

\author{
Adrien Locatelli \\ Lycée les Eaux Claires, 38000, Grenoble, France \\ adriloc38@gmail.com
}

\begin{abstract}
This article relates the first injection in a human being of macromolecules whose primary structure was developed from a religious text.
\end{abstract}

\section{Introduction}

DNA and proteins are macromolecules having a primary structure which can be written with letters. Recent studies have reported that it is possible to convert any type of information into DNA for the purpose of storage. ${ }^{1},{ }^{2},{ }^{3}$ Since it is possible to convert digital information into DNA, I wondered whether it would be possible to convert a religious text into DNA and to inject it in a living being.

\section{Methods}

The Book of Genesis was downloaded from www.fourmilab.ch (https://www.fourmilab.ch/etexts/www/hebrew/Bible/Genesis.html). I replaced each of the Hebrew letters with the nucleotide corresponding to it in the Table 1. Punctuation and spaces are ignored. By applying this method to the first part of the Book of Genesis (Gn1,1 to Gn11,9 excluding Gn2,10 to 2,14, Gn5 and Gn7,1 to 7,5 because these are controversial passages.), I obtained the sequence DNA 11261.4

The surah Ar-Ra'd was downloaded from holyquran.net (https://holyquran.net/cgi-bin/prepare.pl?ch=13). I deleted all the letters of the surah except Alif, Lam, Mim, Ra and Sad and I replaced each of these Arabic letters with the nucleotide corresponding to it in the Table 2. Punctuation and spaces are ignored. I obtained the sequence DNA1511. ${ }^{5}$

I then used the Translate tool from expasy.org on DNA11261. I assembled all the Open reading frames highlighted in red from 5'3' Frame 1 with more than 5 amino acids and I obtained the protein MI518. ${ }^{6}$ I then implanted this protein in a rAAV : VB180513-1026kbp (https://en.vectorbuilder.com/vector/VB180513-1026kbp.html). I then injected $5 * 10^{8}$ genome copies of this viral vector in my left thigh by subcutaneous injection. I then used the Translate tool from expasy.org on DNA1511. I assembled all the Open reading frames highlighted in red from 5'3' Frame 1 with more than 5 amino acids and I obtained the protein : >MT59 MSRERRVESEEEHDRYMRGRNENDRRDECVEKCSGTRTTTEERSATERRETRRMTREAT. I noticed that this protein is very small compared to MI518 and that it contains 2 and only 2 cysteines. I synthesized the central part of this peptide with a disulfide bond connecting the 2 cysteines : >RS27 12-16 RGRNENDRRDECVEKCSGTRTTTEERS. I then injected $7 \mathrm{mg}$ of RS27 12-16 purity 55,17\% raised by Proteogenix in my right thigh by subcutaneous injection.

\section{Results}

The subcutaneous injection of the vector VB180513-1026kbp in my left thigh only caused at the injection site a minor inflammation which persisted a few day and the subcutaneous injection of the peptide RS27 12-16 in my right thigh provoked nothing.

\section{Discussion}

It is the first time that someone injects himself macromolecules developed from a text. It is very symbolic even if it does not have much interest. 


\section{References}

1. Erlich, Y. \& Zielinski, D. Dna fountain enables a robust and efficient storage architecture. Science 355, 950-954, DOI: https://doi.org/10.1126/science.aaj2038 (2017).

2. Church, G. M., Gao, Y. \& Kosuri, S. Next-generation digital information storage in dna. Science 337, 1628, DOI: https://doi.org/10.1126/science.1226355 (2012).

3. Goldman, N. et al. Towards practical, high-capacity, low-maintenance information storage in synthesized dna. Nature 494, 77-80, DOI: https://doi.org/10.1038/nature11875 (2013).

4. Locatelli, A. Dna11261. figshare https://doi.org/10.6084/m9.figshare.7235174.v1 (2018).

5. Locatelli, A. Dna1511. figshare https://doi.org/10.6084/m9.figshare.7235189.v1 (2018).

6. Locatelli, A. Mi518. figshare https://doi.org/10.6084/m9.figshare.7235540.v1 (2018).

\section{Acknowledgements}

I thank www.fourmilab.ch and holyquran.net.

\section{Author contributions}

I did everything in this study, alone.

\section{Human Subject Approval}

I am the only subject of this study and I gave my informed consent.

\begin{tabular}{|l|c|}
\hline Aleph & G \\
\hline Beth & A \\
\hline Gimel & C \\
\hline Dalet & T \\
\hline He & G \\
\hline Vav & A \\
\hline Zayin & C \\
\hline Het & T \\
\hline Tet & G \\
\hline Yod & A \\
\hline Kaf & C \\
\hline Lamed & T \\
\hline Mem & G \\
\hline Nun & A \\
\hline Samech & C \\
\hline Ayin & T \\
\hline Pe & G \\
\hline Tsadi & A \\
\hline Qof & C \\
\hline Resh & T \\
\hline Shin & G \\
\hline Tav & A \\
\hline
\end{tabular}

Table 1. This table was developed by taking inspiration from the Hebrew alphabet and the DNA codon table. 


\begin{tabular}{|l|l|}
\hline Alif & G \\
\hline Lam & A \\
\hline Mim & C \\
\hline Ra and Sad & T \\
\hline
\end{tabular}

Table 2. This table was developed by taking inspiration from the isolated letters from the Quran. 